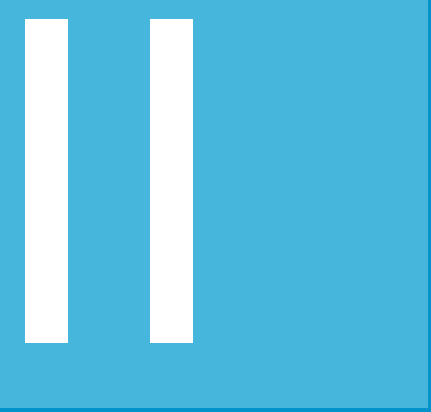

\title{
La cultura organizacional en el contexto de la globalización
}

\section{The organizational culture in the context of globalization}

\section{Universidad de Montemorelos, México}

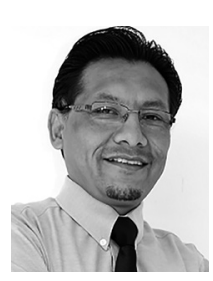

\section{Juan Carlos Niño de Guzmán Miranda}

Licenciado en Administración. Magister en Marketing y Negocios Internacionales y Doctor en Administración. Realizó actividades de docencia y gestión en su alma mater, la Universidad Peruana Unión. Asimismo, es ponente en eventos de índole nacional e internacional. Actualmente se desempeña como docente de pregrado y posgrado de la Facultad de Ciencias Empresariales y Jurídicas de la Universidad de Montemorelos (México).

DOI: http://dx.doi.org/10.17162/au.v0i2.97

Revista Apunt. Univ.

VOLUMEN V • NÚMERO 2 p. 19 - 40 


\section{Resumen}

El objetivo de este artículo es analizar la cultura organizacional dentro del contexto de la globalización económica actual. Para esto, se hace una revisión de conceptos fundamentales como: cultura, cultura organizacional, orientación de las empresas, empresa como agente social, responsabilidad social empresarial y la globalización. Se concluye que las organizaciones tienen el desafío de brindar valor agregado claramente distinguible a sus clientes para que estos ayuden a mantenerlas vigentes.

Palabras clave: Cultura organizacional, globalización, neoliberalismo, responsabilidad social, consumismo

\section{Abstract}

The aim of this paper is to analyze the organizational culture within the context of the current economic globalization. For this, we will a review of fundamental concepts such as: culture, organizational culture and orientation of enterprises, enterprise as social agent, social responsibility and globalization. We conclude that organizations are challenged to provide clearly distinguishable value to their customers so that they can help to keep them in force.

Keywords: Organizational culture, globalization, neoliberalism, social responsibility, consumerism. 


\section{Introducción}

En el contexto de globalización económica, política, social y cultural que se vive actualmente en el orbe se plantea la siguiente cuestión: ¿Cómo se encuentra la cultura organizacional dentro del contexto de la globalización económica actual?

\section{¿Qué es la cultura?}

Al respecto Quintana (2000, p.2) menciona:

Es el fondo sustancial de nuestros pensamientos, de nuestras creencias, de nuestros modos de entender la vida, de nuestras ideologías legítimas...es el conjunto de ideas, valores, normas, gustos, creencias y actitudes que conforman la visión que tiene una sociedad de sí misma y del mundo... de los valores asumidos

Por otro lado Smircich (1983, p. 344) comenta que "es la agrupación específica de normas, estándares y valores compartidos por los miembros de una organización que afectan la forma como desarrolla los negocios". También es considerada como un modelo de suposiciones básicas inventado, descubierto o desarrollado por un grupo, dentro de sus procesos de aprendizaje, para solucionar sus problemas de adaptación externa e integración interna y que han sido trabajadas lo suficiente para ser consideradas como válidas... (Schein, 1988, p. 110).

\section{La Cultura Organizacional}

Algunos conceptos relacionados son:

"Es el repertorio de conductas, la manera de proceder y actuar de una organización en concordancia con los objetivos y metas. Refleja la imagen de la organización. Es como la personalidad de la organización” (Gonzalez, 2009, p. 5). Es el 'alma' de la empresa.

"Es una voluntad de querer ser que identifica a las organizaciones y les otorga personalidad y estilo... se transfiere intangiblemente a los productos y servicios y matiza las relaciones mercantiles..." (Quintana, 2000, P. 3).

"Son el lenguaje, los ritos y los mitos creados y difundidos por ciertos 
directivos para influir sobre los comportamientos de los miembros de la empresa (Pettigrew, 1979, p. 570-581).

"Conjunto de valores, creencias, actitudes, expectativas, racionalidades y aptitudes comunes a todos o por lo menos a la gran mayoría de los miembros de la organización y que influyen, como normas implícitas, en su comportamiento" (Menguzzato y Renau, 1991, p. 22).

El empresario que no tiene cultura empresarial ve a la empresa como su caja fuerte en la que no tiene que velar por el beneficio de los agentes con los que se interrelaciona. Por eso...

- No le importa el desarrollo de su personal

- No le interesan los problemas que ocasiona a sus proveedores

- No le interesa el bienestar del cliente.

El nuevo concepto es que se toma en cuenta a la empresa como un ente al servicio de la sociedad y no como algo que solo beneficia a los propietarios de la empresa (Pinto, 2000, p. 15).

\section{Formación de la Cultura organizacional}

Con la existencia de la cultura empresarial, la conciencia se convierte en factor de producción... sólo que no se compra, ni puede ser solicitada a los suministradores conocidos. Se forma sola, como resultado de hacer, crear y relacionarse; o se forma deliberadamente por la dirección interesada y paradigmática de los directivos... No es posible imponerla (origina servilismo y rebeldía y generan un vacío de valores o una plétora de antivalores). Una verdadera cultura es el resultado de la identificación galbraithiana ("El nuevo estado industrial") de ideales compartidos en un clima propiciador". (Quintana, 2000. P. 3)

\section{Orientación de las Empresas}

Para Quintana (2000), existen empresas de diferentes tipos, según su orientación. Estas son:

\section{- Orientadas a la asimilación del cambio - búsqueda del progreso}

Las empresas orientadas a la asimilación del cambio y la búsqueda constante del progreso, privilegian los valores: 
- Espíritu innovador

- Audacia

- Sentido de oportunidad

Esto crea un clima propicio para el estudio y la superación así como para la investigación y el desarrollo de productos, servicios y procesos.

\section{- Orientadas a ser conservadoras}

No se inclinan al riesgo ni asumen el lucro como misión central de su misión. No aspiran a ejercer liderazgo. Se conforman con mantener un trozo del mercado (clientes fieles). A veces hacen del servicio una experiencia excelente para el cliente y del buen trato un valor sustantivo.

\section{- Orientadas al dinero como inductor de buen comportamiento}

Ven a sus empleados como el HOMO ECONOMICUS. El valor subyacente es el interés económico. Este dará el tono que servirá de pauta al comportamiento general.

\section{- Orientación a buscar motivaciones humanas de afecto}

Existen empresas que promueven las motivaciones de afecto, seguridad o realización.

En el ambiente se difunde un tipo de relaciones basadas en el respeto y el mérito.

\section{- Orientación al orden y la eficiencia}

Ponen a la burocracia como prioridad, al sistema basado en solamente etapa tras etapa.

\section{- Orientación a las estructuras}

También a los métodos orgánicos que promueven la coordinación y cooperación casi espontánea de personas autodirigidas. 


\section{- Seudo-culturas aberrantes}

Existen también empresas con culturas formadas por antivalores y rasgos psicopáticos; culturas de rechazo a los jóvenes (inmadurez) donde se promueve el no respeto por las mujeres (partos, fisiología), la fobia contra los más viejos (¿capital experiencia?), contra religiosos, contra grupos étnicos minoritarios, ex-reclusos... los discursos son tolerantes, pero los individuos que se clasifican en estos grupos son discriminados.

En estos actos de discriminación están coligadas la cultura de la sociedad y la de la empresa. Asimismo, existen culturas que privilegian lo colectivo a ultranza, exacerban el individualismo hasta convertirlo en enfermedad social, culturas democráticas y participativas, culturas totalitarias y excluyentes, culturas del amor, la esperanza y la vida o culturas de la muerte.

\section{La empresa como agente social}

Dolors Oller (2010), opina que en estos tiempos existe la necesidad de plantearse las consecuencias medioambientales y sociales de los modelos de crecimiento económico; el respeto a los Derechos Humanos; el centro de las preocupaciones debería ser la persona humana. El terreno de la ética es el de las convicciones que se plasman en estilos de vida y de actuación (capacidad de autocontrol o autolimitación).

Entonces debe analizarse a la ética nuevamente:

- Ética de responsabilidad: En la que la organización asume las consecuencias de las decisiones tomadas.

- Ética de la convicción: Para discriminar aquellos medios que pervertirán del todo el objetivo de integrar en las decisiones empresariales una visión global para tener responsablemente presente cómo pueden afectar las decisiones tomadas a sociedades cercanas o lejanas.

\section{Responsabilidad Social Empresarial}

La globalización económica ha traído también como tema central para ser tomado en cuenta por las organizaciones a la Responsabilidad Social que estas deben asumir. Oller (2010) resalta la importancia que se debe brindar a la Responsabilidad Social Empresarial (RSE).

Comienza a generalizarse la idea que las empresas no deben ser valoradas únicamente en términos económicos, sino también en términos 
medioambientales y de respeto a los Derechos Humanos. Para ello existen hoy conceptos como la "Triple cuenta de resultados", el sistema "SA 8000", el Índice de sostenibilidad (Dow Jones), entre otros.

Nuestras sociedades son cada vez más sociedades de organizaciones. La empresa como institución socio-económica tiene una importante responsabilidad moral para con la sociedad; por lo tanto, se deben tomar decisiones considerando el ethos. Entonces se debe tener también un enfoque integral de la empresa (tomando en cuenta a los stakeholders).

En estos tiempos la empresa no solamente ha llegado a tener poder económico, sino también poder tecnológico, poder político, poder cultural y hasta poder social.

En este sentido, plantea Ollers (2010) la necesidad de asumir su responsabilidad en todos estos ámbitos dado que no puede pretenderse un comportamiento ético de la empresa si la sociedad que la rodea tiene bajos niveles éticos. A su vez, una cultura empresarial que haya integrado la ética en la gestión de todos sus procesos, será un punto de referencia ético para la sociedad. Que las empresas transnacionales ejercieran el papel de transmisoras de los Derechos Humanos. Que se promueva el respeto a los trabajadores, el respeto a los grupos de interés, el buen ambiente de trabajo, las buenas condiciones laborales, los salarios justos, la formación, la seguridad e higiene ocupacional.

Una muestra de los avances en esta propuesta la puede dar el Parlamento Europeo (1997) que publicó las principales responsabilidad éticas de la empresa con los trabajadores y la comunidad. Entre estas se encuentran:

a) Servir a la sociedad con productos útiles y en condiciones justas.

b) Crear riqueza de la manera más eficaz posible.

c) Respetar los derechos humanos con unas condiciones de trabajo dignas que favorezcan la seguridad y salud laboral y el desarrollo humano y profesional de los trabajadores.

d) Procurar la continuidad de la empresa y, si es posible, lograr un crecimiento razonable.

e) Respetar el medio ambiente evitando en lo posible cualquier tipo de contaminación minimizando la generación de residuos y racionalizando el uso de los recursos naturales y energéticos.

f) Cumplir con rigor las leyes, reglamentos, normas y costumbres, respetando los legítimos contratos y compromisos adquiridos.

g) Procurar la distribución equitativa de la riqueza generada.

h) Seguimiento del cumplimiento de la legislación por parte de la empresa.

Revista Apunt. Univ. VOLUMEN V • NÚMERO 2 p. $19-40$ 
i) Mantenimiento de la ética empresarial y lucha contra la corrupción.

j) Supervisión de las condiciones laborales y de salud de los/as trabajadores.

k) Seguimiento de la gestión de los recursos y los residuos.

I) Revisión de la eficiencia energética de la empresa.

m) Correcto uso del agua.

n) Lucha contra el cambio climático.

o) Evaluación de riesgos ambientales y sociales.

p) Supervisión de la adecuación de la cadena de suministro.

q) Diseño e implementación de estrategias de asociación y colaboración de la empresa.

r) Implicar a los consumidores, comunidades locales y resto de la sociedad.

s) Implicar a los empleados en las buenas prácticas de RSE

t) Marketing y construcción de la reputación corporativa.

Basados en la Santa Biblia, el mejor ejemplo que Jesús brinda acerca de este concepto es con la parábola del Buen Samaritano, en la que el amor es el centro de todo comportamiento, ese amor proveniente de Dios y puesto en práctica en la vida cotidiana del ser humano, más allá de los prejuicios y teorías que pueden sustentar determinadas posturas. Significa que el interés por los semejantes nuestros debe ser genuino y sin buscar algún beneficio particular en desmedro de la otra parte.

\section{La Globalización}

Es el proceso por el cual diversas partes del mundo interactúan económica, política y culturalmente. El intercambio entre diversas culturas empezó hace miles de años. En el siglo XIX explotó el intercambio cultural, los europeos exploraron el continente americano, el imperialismo europeo lideraba en el orbe, se dio paso a la revolución industrial y los inventos más grandes hasta la fecha, se dieron a conocer como el transporte, el teléfono, el telégrafo, entre otros. Ya en el siglo XX, se dio el fin de la guerra fría, dando paso al capitalismo de mercado libre, a la aparición de internet, en la que gana mayor fluidez el intercambio de nuevas ideas, las transferencias de dinero, el intercambio cultural, la disponibilidad de los servicios.

Revisados los conceptos fundamentales para describir la manera en 
que está siendo influenciada la cultura organizacional en este mundo en el que la globalización económica predomina, se ha llegado a las siguientes conclusiones:

\section{En medio de la globalización, la cultura organizacional viene cambiando su postura para centrarse en el consumidor/cliente}

Hoy en día, las empresas locales se han visto obligadas a entrar al ritmo de las de talla mundial, dependiendo, en mayor o menor grado, del mercado al cual atienden; esto va acompañado del cambio de postura del Estado (como institución) que lejos de ser el ente preocupado solamente en cobrar impuestos, debe incentivar la mayor competitividad de las empresas.

Hoy las guerras se hacen con armas de gran alcance, pero también con la economía. Existen muchos gobiernos que tienen como estrategia el empleo del marketing internacional para brindar una plataforma en conjunto, de propuestas de productos o servicios a nivel internacional. Quiere decir que los Estados, se ven en la necesidad de impulsar la generación de empresas que consigan ocupar mercados internacionales, esto genera un incremento de divisas para el país, mayores impuestos, mayor rotación del capital invertido en el país, generación de empleo, entre otros beneficios.

En este sentido, tienen que prepararse las empresas para competir y el Estado, garantizarle condiciones para que pueda competir ante empresas nacionales y extranjeras. El más competitivo gana una porción del mercado por un tiempo.

Ya las empresas no se pueden dar el lujo de fabricar productos simplemente por fabricar, es el mercado que le aceptará o no la oferta presentada. Entonces, la empresa tiene que indagar qué es lo que el mercado necesita, para recién empezar a producir aquello que precisa. Esa indagación la realiza al cliente, al consumidor, al usuario, al ciudadano que forma parte de un segmento de la población. Este cliente se ha convertido en el centro de atención en los negocios. Ya no lo es el producto.

Bajo este criterio, se inserta el concepto de competencias para laborar en la empresa de acuerdo a la necesidad de mano de obra calificada que se tenga en la misma.

Hoy la cultura organizacional está mudando sus prioridades, dando paso a la competitividad para elaborar productos o brindar servicios de calidad que satisfagan a ese cliente 'voraz' que tiene capacidad de pago y que cada vez más, aprovechando esa condición de "rey" que le ha otorgado la economía de libre competencia, exige mayores beneficios al menor precio posible. 


\section{El avance científico y tecnológico proporcionó al ser humano la oportunidad de "romper las fronteras", entre los países y entre los continentes. Esto ha generado una priorización de tener mano de obra calificada en la organización}

Muchas sociedades no podían contar con algunas ventajas de la tecnología ni los beneficios del avance de la ciencia.

La competencia generada por esta globalización, cuyo nacimiento no fue en el siglo XX, sino con mucha anterioridad entre los asiáticos, árabes y otros pueblos vecinos, hizo que los países, que se insertaron en este mercado abierto, impulsaran a las empresas a internacionalizarse. Se empezó a atender a la demanda interna primero y luego a ingresar a mercados vecinos para posteriormente sumergirse en esa batalla por los mercados de diversas latitudes.

Los países en vías de desarrollo, cuya población se ha visto postergada por muchas décadas, ahora pueden acceder al servicio de algunos inventos y de tecnología de punta a costos muy reducidos, esto porque las empresas, sumergidas en la competencia, han descubierto que el grueso del mercado, que es ese ejército de consumidores, a la larga suman grandes cantidades de dinero pagando precios accesibles para el uso de tales adelantos tecnológicos.

Se pueden constatar ejemplos de la telefonía celular, la internet, la televisión por cable, artefactos que han contribuido a solucionar problemas de distancia, salud, entre otros. Estos productos, en su mayoría de origen chino u otro país asiático, son de costos bajos, debido a las economías de escala que utilizan para su producción y la reducción de costos por las compras gigantes de insumos, a esto se agrega la explotación de la mano de obra que tiene un mínimo costo debido a las políticas gubernamentales aplicadas, algunas veces, autoritariamente.

Las distancias se han acortado con toda esta tecnología, uno de los beneficiarios de este fenómeno ha sido de todos modos el cliente, poblador de estos países pobres y en vías de desarrollo.

Sin embargo, otro grupo de beneficiados, muy reducido, y por ende con grandes ganancias, han sido los inversionistas. Quienes, poniendo capital, han podido obtener grandes ganancias.

En esta explosión del consumo, generada por una corriente que tiene su sustento en el posmodernismo, se observa que ha calado hondo el uso de la tecnología. Esto a la vez obliga a mucha gente a conocer y dominar las Tecnologías de Información y Comunicación (TICS) para poder ser competitiva en el mercado laboral.Se ha podido observar que grandes cantidades de pobres han empezado a salir de esa condición gracias a las facilidades que brinda la tecnología, fundamentalmente las comunicaciones. 
Esto viene influyendo en la cultura organizacional de muchos países, porque en vista que una de las prioridades radica en abaratar costos y siendo que la mano de obra es un factor preponderante de estos costos, en algunos casos se abusa de esta mano de obra. Por el exceso de oferta de ésta y por la baja calificación que tiene la misma. A esto se agrega la alta cuota de trabajadores que desconocen la totalidad de sus derechos laborales o a veces, incluso conociéndolos, no los hacen respetar debido a la excesiva competencia de mano de obra barata y la necesidad de llevar dinero a casa para cubrir los gastos familiares básicos y cotidianos.

No obstante, existen algunas empresas que, en pos de obtener certificaciones internacionales de calidad y mirando el largo plazo, en lugar de abusar de esa mano de obra no calificada, prioriza el buen trato a ellos, capacitándolos, buscando su desarrollo personal. Siempre que hayan pasado estos por un proceso riguroso de selección, de tal forma que invierten en personas valiosas realmente para generar un valor agregado en los productos o servicios que ofertan luego al mercado.

3. La lógica que subyace a las políticas educativas difundidas por el neoliberalismo es la lógica del negocio

Los valores que proclama son la eficiencia como el camino hacia la competitividad, y la productividad es el beneficio como recompensa y fines a ser alcanzados.

Aquí existe una disyuntiva, ¿̇uál de las dos priorizar?:

a) ¿El centro educativo, que tiene que ser bien gestionado para lograr sus objetivos (económicos principalmente)? o

b) ¿El centro educativo, como centro de formación de personas, ciudadanos útiles a la sociedad?

No es posible educar por educar, es decir brindar una seudo educación que sirva para enriquecer a la persona o personas que invirtieron un capital simplemente. Hay escuelas, colegios, institutos y universidades que priorizan solamente el tema económico para mantenerse en el mercado, exponiendo con esto una desfachatez pues lo que han logrado es ofrecer seudo profesionales al mercado laboral. Esa puede ser una de las causas de la debacle moral de algunos países que tienen esta condición.

Todo esto no es una cosa casual. Se ha convertido a las personas a

Revista Apunt. Univ. VOLUMEN V • NÚMERO 2 p. $19-40$ 
ser meros consumidores, buscadores de placeres y personas que quieren vivir el momento, sin evaluar consecuencias... "total, a mí no me afecta" esa es la lógica individualista a la que con lástima se está llevando a muchos pueblos.

El país que tiene esta coyuntura necesita cambios en este sentido. La educación es fundamental para que el ciudadano tenga mejor criterio para elegir. Para que tome decisiones basadas en valores. El Estado juega un rol importante en este aspecto, puesto que debe invertir sin buscar retorno al corto plazo. Le conviene tener un ciudadano más culto, mejor informado, que sea consciente de vivir buscando el bien común. En este aspecto, el neoliberalismo afecta de todos modos al sistema educativo por priorizar el tema comercial en algo tan importante e invalorable para una comunidad: la educación de sus habitantes.

Las organizaciones, de alguna manera, son afectadas por esta lógica en el sentido que toda labor que un colaborador debe realizar corre el riesgo de ser confundido como un favor extra y que merece ser recompensado (muchas veces material o monetariamente) por aquel que recibió dicho servicio dentro de la misma organización; generando de esta forma vicios que acaban en corrupción y mal uso de recursos. Todo por obedecer a la lógica del negocio. Por lo tanto, es preciso considerar la labor importante que realizan los líderes en la organización para prevenir estos casos.

\section{El liberalismo económico es un sistema que promueve la productividad y la competitividad; no obstante, a las finales alguien gana y alguien pierde}

La persona, tiene derecho a decidir en un ambiente de libertad, con esto decide si se hace competitivo y/o productivo o simplemente si no "obedece" este sistema y se dedica a otras cosas sin importarle de dónde vengan los medios para sobrevivir.

En este sentido, si se compara a un poblador de un sistema económico comunista con uno que vive en un sistema económico de mercado abierto. Resulta que el primero resulta mucho más pasivo, sin afán de progresar, esperando el asistencialismo y siempre pasivo, con la esperanza de que el gobierno en algún momento vaya a hacer algo por ese ciudadano.

Por otro lado, particularmente en el Perú, personas dedicadas al comercio han sido testigos de cómo en medio de todos estos cambios de paradigmas, de los golpes de Estado, de los cambios de gobierno con inclinaciones a la izquierda o a la derecha, quienes han sabido sobrevivir y todavía permanecen en pie, tal vez sin interesarles quién gobierna incluso; estos son los micro y pequeños empresarios (mype). 
El micro o pequeño empresario, tal vez sin formación profesional, ha aprendido a mantenerse atento a la realidad, a aprovechar oportunidades, a perseverar, a postergar placeres cortoplacistas, a conocer a sus clientes ("caseros"). Ha aprendido a buscar la calidad, no porque le dictaron una asignatura con especialistas de talla internacional, sino con la experiencia, cayendo en varios intentos tal vez. Ha sido víctima de muchos ataques de los gobiernos de turno, locales, regionales o nacionales. Pero ha sabido sobrevivir. Es pues, un ciudadano que no se preocupa por la autoridad que salió elegida, sino por cómo tendrá que actuar en cada nuevo escenario que se le presente.

Con este ejemplo del micro o pequeño empresario, se pretende demostrar de cómo es que la prioridad ahora es la productividad y la competitividad. Dada la competencia dentro y fuera de las empresas, hoy existe una cultura que promueve ser el mejor, exacerbando el individualismo. La forma de competir está dada: "hay que ser productivo y eficiente". Lamentablemente dentro de este modelo el que es más débil tiene grandes posibilidades de perder en esta competencia. Significa que se requiere de un mínimo de preocupación por el otro, por el que pierde en la competencia. Para ello se deben instaurar "reglas claras de juego", con instituciones que respeten y hagan respetarlas; por ende, con personas dignas de confianza que el Estado, autoridades y ciudadanos confieren.

Conociendo la naturaleza humana, no se puede asegurar que funcione correctamente esa recomendación. Sin embargo, existen salvedades como las personas que practican principios y toman decisiones basadas en sus valores claramente fundamentados. Personas justas que valoran al ser humano como tal.

\section{El papel de los centros educativos debe ser el de formar personas formadas integralmente y hacer de estos el lugar donde surge la producción, la apro- piación y la socialización del saber}

Si bien es cierto que "la verdadera educación significa más que la prosecución de un determinado curso de estudio... es el desarrollo armonioso de las facultades físicas mentales y espirituales." (White, 2013, p. 13); de acuerdo con Saviani (2010, p. 117-133), la escuela tiene una función específica, educativa, propiamente pedagógica, ligada a la cuestión del conocimiento; es preciso rescatar la importancia de la escuela, y reorganizar el trabajo del proyecto nacional de la educación.

Los centros educativos, sea cualquiera la forma de sistema de gobierno que adopte un país, deben ser aquellos donde surge la producción, la

Revista Apunt. Univ. VOLUMEN V • NÚMERO 2 p. $19-40$ 
apropiación y la socialización del saber. El ciudadano tiene todo el derecho de recibir ese saber, aprender a manejarlo y compartirlo; pero también el deber de producir mayor conocimiento (saber), haciendo una contribución a la sociedad. Esto porque se necesita tener ciudadanos más inteligentes, que tengan la libertad de decidir con suficiente fundamento.

Por un lado, alarma pensar que la aristocracia propone que la educación sea solo dirigida para las élites que ellos deciden, que buscan consumidores sometidos y esclavizados a ganar un cúmulo de dinero suficiente para mantenerse vivos y continuar en este sistema. En otras palabras que sean como máquinas consumidoras que no piensen y que tomen sus decisiones en base a suposiciones, percepciones superfluas o a simples "corazonadas". Esta manera de pensar lastimosamente invade cada vez más a empresarios y comerciantes.

Por otro lado, existe la otra corriente que, en los centros de educación, se ponga énfasis en la reproducción de una sociedad que permita distribuir la riqueza por igual. Si bien es cierto, tiene bases loables que resaltan la igualdad, no necesariamente es practicada por los ideólogos y las cúpulas que promueven esta corriente de pensamiento, confundiendo las mentes de las personas que lo que van a recibir como formación en los centros de enseñanza son competencias que permitan preparar a ese ciudadano y no solamente inclinaciones político-sociales.

En este sentido, ni el neoliberalismo, dando rienda suelta a esa libertad que pregona, tiene formas claras de controlar esas maneras parcializadas de actuar ni el socialismo, que cuando llega al poder no sabe cómo gestionar, llegan a convencer.

Lo que es cierto es que la cultura organizacional que tenga elementos con extremismos liberales o socialistas no sale beneficiada si es que la intención no toma en cuenta a la persona como eje del trabajo que se vaya a realizar. Esta persona es la llamada a utilizar sus conocimientos, adquiridos en los centros de enseñanza como también aprendidos en las experiencias que haya obtenido, para bien de la sociedad.

\section{Los beneficios de vivir activamente en la globalización van orientados para pocos en perjuicio de muchos otros}

En vista que el neoliberalismo ha empujado a pueblos enteros a adoptar nuevas reglas de juego, el espíritu de competencia ha salido a relucir. Entonces, existe el afán de obtener beneficios máximos de todos los recursos que sean posibles, incluyendo de las personas comunes y corrientes. 
Hay una premisa: "para ganar hay que arriesgar". En la medida que se invierta más dinero, tiempo, personas, el inversionista espera recibir más. Esta lógica de los negocios puede ser válida en algunos escenarios. No obstante, ha superado esas barreras y existen empresarios que se pueden prestar para comprar consciencias.

Muchas personas han invertido en negocios, esperando obtener ganancias legítimas; sin embargo, si se compara el número de beneficiarios (nuevos ricos) con el número de las personas que directa e indirectamente han participado para contribuir en esa acumulación de riqueza (elementos de producción), se tiene el resultado que el número de más ricos es menor que el de los más pobres.

Por consiguiente, hay una desigualdad que va acrecentando; cuando la lógica comúnmente nos dice que el que se esfuerza triunfa, pero hay muchos trabajadores que se esfuerzan y no ven resultados al cabo de algún tiempo.

Viene la incógnita, por qué es que triunfan uno y otros no. La respuesta puede estar en la diferencia de estrategias que han utilizado para triunfar.

Significa que hay que tener una visión más aguda de cómo llegar a ello, tal vez especializándose en determinadas ramas y no haciendo lo mismo que los demás, tal vez, estudiando a la competencia, tal vez, analizando internamente en qué cosas se está marchando equivocadamente, o tal vez invirtiendo más en capacitación, manejo de información y nuevos conocimientos, que es lo que brinda mayor valor agregado a las economías de países que necesitan crecer actualmente.

El hecho es que lamentablemente, en este juego de todos modos van a existir ganadores y perdedores.

\section{La globalización es un fenómeno que conlleva perjuicios al medio ambiente, por lo que las empresas deben tomar en cuenta este problema}

Actualmente el medio ambiente, en todas sus connotaciones, está siendo afectado por diversas empresas, en algunos casos con la anuencia del Estado y en otros con la omisión de su presencia.

La emisión de gases tóxicos provocada por industrias que irresponsablemente afectan la capa de ozono no puede ser controlada por ningún tipo de organismo nacional o internacional. La Organización de las Naciones Unidas, a través de la Convención Marco de las Naciones Unidas sobre el Cambio Climático puesta en vigencia a partir de 1994, busca llamar a la conciencia pública a nivel mundial acerca de los problemas relacionados a este asunto. A partir de 1997

Revista Apunt. Univ. VOLUMEN V • NÚMERO 2 p. $19-40$ 
con la firma del Protocolo de Kyoto, se implementan medidas más enérgicas para el cuidado del medio ambiente. El objetivo radica en la estabilización de concentraciones de gases de efecto invernadero. En Nairobi (2006), Copenhague (2009), México (2010), Durban (2011), y recientemente Lima (2014) se han hecho algunas enmiendas, sin embargo, algunas potencias económicas no desean ratificar este protocolo que forma parte de este propósito porque no le conviene, puesto que un gran número de industrias está provocando estas concentraciones de gases de efecto invernadero, uno de sus pretextos es que exige que los países emergentes, como China, se sumen a la firma y respeto de estos términos.

Algunas Organizaciones No Gubernamentales (ONGs) se están dedicando a este fin; no obstante, no es suficiente.Entonces, a todo esto, la cultura organizacional tiene un rol por desempeñar frente a este problema. Influye mucho la decisión de los líderes que laboran en una organización, puesto que de una u otra manera, podrán inducir a que los demás trabajadores tomen conciencia primero, y adopten una actitud de respeto al medio ambiente.

El desperdicio inconsciente y egoísta de algunos recursos naturales como el agua, los combustibles, árboles, energía eléctrica; está provocando problemas de todo tamaño simplemente porque no se está respetando los derechos de otros. Problemas de salud pública, males congénitos, cambios bruscos en el clima, muertes de animales y otros problemas que se generan de estos otros, vienen siendo parte de la vida común de los habitantes del planeta en estos tiempos.

Un cristiano, comparte su creencia, y, como tal, acepta a Dios como creador de toda la naturaleza y del hombre. En este sentido, respeta también todo lo creado por Dios. No por algún interés banal, ni porque le obligan o porque va a recibir un castigo; sino porque respeta a su prójimo y a sí mismo. La solución de este problema entonces parte por lo individual.

En la globalización, la cultura organizacional viene siendo presionada para practicar hábitos que dañan el medio ambiente, no reciclando desperdicios y abusando del uso de recursos para su desempeño laboral. El neoliberalismo, no hace hincapié en este aspecto puesto que, de alguna manera, resalta el individualismo y éste muchas veces ignora el bien para otros, normalmente los menos informados, por ende menos favorecidos. 


\section{La migración de grandes grupos de personas amenaza la identidad de los pueblos y la poca calificación de estas impide que lleguen a tener niveles de vida adecuados}

Con la globalización de la economía, se ha dado el fenómeno de la migración y con esto se ha puesto en amenaza latente de desaparición a algunos pueblos autóctonos que tienen una cultura muy rica en costumbres, en una cosmovisión diferente de la vida y que pueden aportar mucho a la sociedad. Esa identidad bien fundamentada de la que muchos pueblos carecen y ese amor a la tierra que los vio nacer, se puede percibir en las culturas autóctonas.

Siendo que son minorías y que no tienen suficiente fuerza para hacer escuchar sus propuestas, o sus necesidades, y si a esto se suma la migración de sus habitantes a otras latitudes, adquiriendo hábitos y costumbres citadinas, lamentablemente existe el riesgo de la extinción de tales culturas.

Pueblos oriundos de la selva y/o la sierra están desapareciendo producto de la carencia de servicios, de la apertura comercial que se ha dado en ciudades de la costa o capitales de regiones, en vista de esas comodidades y la modernidad que ha llegado a tales ciudades, ven por conveniente adoptar nuevas costumbres, nuevos hábitos de consumo, se adaptan a otro sistema de vida, que prioriza el hedonismo, la vida superflua, el consumismo basado en las emociones, dejando de lado sus formas de vida que practicaban con sus familias en sus pueblos originarios.

Otro fenómeno que contribuyó a este problema fue, en el caso del Perú, la aparición del terrorismo, ahuyentando a los habitantes de estos pueblos, y esto generó una invasión de ciudades, sobre todo de la costa. Gente que sin tener una economía estable suficiente para cubrir gastos elementales de sobrevivencia, se vio en la necesidad de comenzar de cero, de aprender a vivir en las grandes "selvas de cemento", donde no tienen la libertad de vivir en medio de la naturaleza ni la facilidad de tener alimentos frescos cerca de casa.

El terrorismo hizo desaparecer poblaciones enteras haciendo uso de armas y proponiendo ideas que no promueven necesariamente las soluciones inmediatas y tangibles para estos pueblos. En muchos de estos pueblos, se desarrollaron enfrentamientos armados, creando confusión y zozobra, que generó a la vez una autoestima muy baja en medio de sus pobladores.

Muchos de esos migrantes, hoy son parte de la masa laboral de diferentes tamaños de empresas. Algunos eligieron capacitarse, educarse y ser profesionales, algunos eligieron aprender a producir algo para sobrevivir y otros simplemente migraron para buscar otros tipos de vida, confundiéndose entre 
gente que busca vivir de la manera que se pueda. Ha perdido esa identidad, se ha convertido en un poblador más que lucha por sobrevivir en medio de la competencia feroz de todo tipo que se vive en las ciudades.

Algunas empresas, viendo nuevas posibilidades económicas, decidieron ingresar a estas grandes extensiones de terreno, abandonadas por los primeros propietarios, en busca de recursos naturales útiles para la minería, hidrocarburos, combustibles o de sembrar en grandes extensiones de tierra algunas semillas de origen transgénico. La biodiversidad de estas zonas geográficas está siendo amenazada, además de la desaparición de estos pueblos autóctonos.

En este sentido, la cultura organizacional de algunas empresas actuales se ha visto obligada a cambiar de paradigmas, vale la explotación a cambio de la generación de ganancias que no siempre van en beneficio de los pueblos donde se explotan tales recursos. La ley permite que se explote solo lo suficiente, sin embargo, muchas empresas han hecho caso omiso a tales reglas, excediéndose en algunos casos y abusando de los derechos que le competen.

En medio de esta realidad, existen grupos políticos, aprovechando la ignorancia de la gente originaria de esos lugares, que se prestan para organizar levantamientos con fines meramente políticos, utilizando a muchos de sus pobladores para generar violencia y caos, bajo el supuesto de que necesitan "libertad" para elegir su destino, bloquean carreteras, incendian propiedades ajenas, protestan sin muchas veces saber los móviles o las causas reales de su protesta. Eso no es para nada una causa justa.

9. La producción de alimentos transgénicos puede ser una amenaza para la salud pública de muchos pueblos, sobre todo aquellos que no pueden pagar altos precios por alimentos de calidad para solucionar el hambre

En vista de la prioridad que ha puesto la ONU como "Objetivos de Desarrollo del Milenio", el primero de ellos es "Erradicar la pobreza extrema y el hambre", algunas empresas han aprovechado para justificar sus prácticas de la producción de alimentos genéticamente modificados consiguiendo grandes ganancias sin priorizar la salud de los consumidores de tales alimentos debido a que representan una amenaza real y potencial dado a que no existen pruebas contundentes de los daños que estos podrían causar.

Estos alimentos normalmente son producidos en diversas partes del mundo, como por ejemplo en Estados Unidos, Brasil, Argentina, India y Canadá, existen leyes que avalan este tipo de agricultura. Son vendidos en casi todos 
los mercados de alimentos del mundo. Pocas son las empresas que manejan estos gigantescos negocios y sus precios son mucho más bajos en comparación con los producidos de manera natural u orgánica. Estos productos tienen mayor demanda en los mercados que no pueden pagar precios altos. Quiere decir que los países pobres o aquellos en vías de desarrollo se ven tentados a importar tales alimentos.

El problema viene en las consecuencias irreversibles que pueden causar estos alimentos genéticamente modificados. Aun no existen pruebas fehacientes que demuestren lo contrario. No obstante, ya se pueden encontrar algunas investigaciones que advierten esto, como el caso de Xue, Yang, Liu y Xue (2012, p. 314-318).

La ambición de estos empresarios ha rebasado los límites, puesto que están jugando con los destinos de ingentes cantidades de personas. Lamentablemente existen gobiernos que permiten estas prácticas, ¿qué intereses hay detrás de estos?

Esta globalización, en este sentido, está empujando a una cultura organizacional que no respeta los derechos de los ciudadanos; sin sentido de equidad, justicia ni preocupación por el prójimo.

Los líderes de tales empresas son los llamados a meditar seriamente estos asuntos puesto que son ellos quienes tomarán decisiones que afectarán muchas vidas humanas. Quiere decir que la ética es un elemento importante que no debe faltar a la hora de tomar decisiones.

Tesis 10: La cosmovisión de muchos trabajadores se ha tornado en consumista y esto ha generado una sociedad que prioriza el culto al egocentrismo y al hedonismo

Mientras se termina la elaboración de este documento, existen miles de personas que emprenden el retorno a casa, otras empiezan a laborar en un segundo o tercer centro de labor, otras están celebrando algo en algún centro de diversiones, de relajo o de ocio, otros están en plena capacitación para aprender a ser más eficientes en el uso de sus recursos y por ende para incrementar sus ingresos, otros están viajando en el bus o tren que toma dos o tres horas en llegar a casa, aquel lugar donde solo llegan a descansar unas cuantas horas y que más parece un hotel que un hogar. Estos son algunos ejemplos de lo que se vive en la gran ciudad, aquella que no descansa en ningún momento. Esta ciudad se ha convertido en el sueño de muchos migrantes y en la pesadilla de otros que no ven la hora de salir de entre sus calles para respirar un poco de aire puro y "desconectarse" de la realidad que los agobia.

Revista Apunt. Univ. VOLUMEN V • NÚMERO 2 p. $19-40$ 
Existen hombres que gustan de hablar sobre las marcas más exitosas de automóviles, de equipos de tecnología de punta que están utilizando, en algunos casos no tienen muchos fondos para alimentarse saludablemente y priorizan la compra de ropa o accesorios que los ayudarán a mantener la apariencia que exige la sociedad. Buscan ese vestir elegante para encontrar a aquellas mujeres, que desean "pasarla bien", no mirando el futuro de corto ni largo plazo.

Existen mujeres que hoy priorizan también el buen vestir, buscan como pareja a alguien que pueda costear sus gastos en moda, estética, cirugías, son de mirada esquiva y falso reír, hablan solamente con aquellos que son de su estatus social.

Existen parejas cuyo jefe de familia está pensando solamente en dinero debido a los gastos que se hizo y que hay que pagar pronto con aquella tarjeta de crédito que ya está sobregirada por haber abusado en la satisfacción de algunas necesidades y otros objetos que fueron comprados solo por deseo banal, dinero para pagar también los gastos de la esposa que ha hecho una "gran inversión" en una operación de nariz o cirugía de rejuvenecimiento facial y que no se sabe si es para "mantenerse más atractiva para su pareja" o para "mantenerse vigente ante la 'competencia' que encuentra a cada paso".

Hay algunas familias que tienen hijos pequeños y a quienes se les prohíbe hablar con niños de color extraño. Familias que aparentan lo que no son, viviendo en un mundo de pura ilusión, ahogados en deudas para mantener su estatus social.

Existen ciudades enteras pobladas de gente consumista, saturadas de edificios y la polución campante, ciudades que cuando suceden accidentes de tránsito, muertes, dolor, nadie llora ni ríe, ciudades frías con personas que tienen semblantes que oyen pero no escuchan y ven pero no miran. Lamentablemente existen sociedades que han adoptado estas posturas ante el modelo capitalista, algunos que pueden vivir de la manera que se describe y otros simplemente anhelando vivir así.

Lipovetsky (1993, p. 20-21), resume este punto de vista: “El mundo del trabajo es el que ofrece la mayor resistencia a la lógica de la seducción, a pesar de las revoluciones tecnológicas en curso. Sin embargo la tendencia a la personalización se manifiesta en él... hoy cada cual es un agente libre de su tiempo, menos sujeto a las normas de las organizaciones rígidas, la seducción es privática... la última moda es la diferencia, la fantasía, el relajamiento; lo estándar, la rigidez, ya no tienen buena prensa. El culto a la espontaneidad y la cultura psi estimula a ser "más" uno mismo, a "sentir", a analizarse, a librarse de roles y "complejos". 


\section{Propuestas}

La sugestión a la que han llegado a ser víctimas muchos pueblos, sobre todo aquellos en los que habitan personas con menores recursos se ha convertido en una amenaza para construir una identidad, un amor genuino por la organización donde uno trabaja o la ciudad donde uno vive. Entonces, se enumeran algunas propuestas para mejorar esta condición que afecta a la organización, consecuentemente, a la sociedad:

- Educar al trabajador o colaborador para que aprenda a pensar, ordenar la información disponible y tomar, con buen criterio, decisiones que tendrán consecuencias en el futuro; así como asumir las consecuencias de tales decisiones.

- Indagar nuevas formas de cambio siempre respetando los derechos de las personas.

- Diseñar un modelo basado en la igualdad para beneficiar a todos de manera justa.

- Buscar sabiduría en otras fuentes para evaluar nuevas alternativas.

- Diseñar estrategias, en las organizaciones, para brindar valor agregado real en los productos o servicios que se brinda a los consumidores como por ejemplo información útil y veraz para mejorar la calidad de vida de estos.

Se puede enumerar muchas alternativas más. No obstante, se debe tener en cuenta que este mundo, a través de los años, ha probado muchas fórmulas, modelos y procesos para mejorar los niveles de vida de sus pobladores. Han probado soluciones en base a causas que tal vez no han sido analizadas en una dimensión más completa.

La propuesta final del autor radica en analizar al ser humano como tal, quien por naturaleza tiende a ocuparse solamente en sus propios problemas y por ende alimenta más su ego.

En busca de satisfacer esas necesidades y sobrepasar esos deseos que se le presenta al hombre, éste se ha atrevido a diseñar estrategias, desde las más simples hasta las más complejas, para llegar a acumular riqueza material, el mismo que habiendo pagado todos esos gastos para satisfacer tales necesidades o deseos, ha crecido tanto, en algunos casos, que en todo el tiempo de vida que le quede no va a poder ser capaz de gastarlo. Este llega a ser un exceso. Estos excesos tienen consecuencias, el ser humano toma decisiones, muchas veces, sin importarle incluso la vida de otro ser humano. Estas consecuencias 
involucran a otras personas que pueden o no ser responsables directas, pero a las finales pueden ser víctima de tales consecuencias.

Dios, a través de la Santa Biblia, menciona que se haga a otros lo que quisiera uno que se haga con él (Mateo 7:12). Una enseñanza tan sencilla que ayudaría mucho a vivir en paz entre la gente. El hombre es un ser tan complejo, de quien no se puede abordar sus problemas solamente bajo un enfoque. Se necesita encontrar otras respuestas. Queda la tarea entonces de indagar más acerca de este ser humano en la fuente de aquel que lo diseñó y lo creó: Dios.

Juan Carlos Niño De Guzmán Miranda

Universidad de Montemorelos, México email: jcnino@um.edu.mx

Recibido: 20 de marzo de 2015 Aceptado: 25 de junio de 2015

\section{Referencias}

Dermeval, S. (2010). Modelos de desarrollo y estilos educacionales en el proceso de emancipación de América latina. Historia de la educación: Revista interuniversitaria, 29 (1). pp. 117-133.

Gonzalez Sterling, L., Hurtado Henao V. (2009). Diagnóstico de la cultura organizacional en la empresa Cointelco. Tesis no publicada. Universidad de la Sabana. Colombia.

Lipovetsky, G. (1993). La era del vacío. Anagrama, Barcelona. P. 20-21.

Menguzzato, M. y Renau, J. (1991). Dirección estratégica de la empresa. Madrid: Editorial Ariel Económica.

Oller Sala, Dolors. (2010). Cambio de paradigma empresarial: la empresa como agente social. http://www.emprendices.co/cambio-de-paradigma-empresarial-la-empresa-comoagente-social/ España.

Pettigrew, Andrew. (1979). Qualitative Methodology Administrative Science Quarterly, Vol. 24, No. 4. pp. 570-581.

Pinto Castro, J. F. (2000). La culta empresaria y los planes de desarrollo de la empresa. Revista de la Facultad de Ciencias Económicas. UNMSM.

Quintana, J. (2000). Cultura empresarial. Revista Vitral http://www.vitral.org/vitral/vitral39/ econom.htm

Santa Biblia (1960). Versión Reina-Valera. Edit. Broadman \& Holman.

Schein, E.H. (1990). Organizational Culture. American Psychologist. P. 109-119.

Smircich, L. (1983). Concepts of culture and organizational analyses. Administrative Science Quarterly 28. 339-358.

White, Ellen (2013). La Educación. México: Inter-American Division Publishing Association. GEMA Editores.

Xue Kun, Jing Yang, Biao Liu, Dayuan Xue (2012). The integrated risk assessment of transgenic rice Oryza sativa: A comparative proteomics approach. Food Chemistry, Volume 135, Issue 1(1). p. 314-318. 